\title{
Stochastic Color Interpolation for Digital Cameras
}

\author{
Hung-An Chang and Homer H. Chen, Fellow, IEEE
}

\begin{abstract}
This paper presents a stochastic estimation approach to adaptive interpolation of color filter array. It models an image as a 2-D locally stationary Gaussian process and achieves robustness against aliasing by employing an edge-sensitive weighting policy based on the stochastic characteristics of uniformly oriented edge indicators. Experimental results show that the algorithm can effectively eliminate the occurrence of perceptible artifact. Performance comparison in terms of peak signal-to-noise ratio and mean square error is provided to demonstrate the superiority of the proposed algorithm.
\end{abstract}

Index Terms-Color filter array (CFA), color interpolation, demosaicing, edge detection, edge-sensitive weighting.

\section{INTRODUCTION}

$\mathbf{M}$ OST digital cameras with a single CCD or CMOS sensor array use a color filter array (CFA) to capture images. The most commonly used CFA is the Bayer color array, or Bayer pattern [1], that samples the blue and green colors alternatively in the even rows and the green and red colors in the odd rows as shown in Fig. 1, resulting in a subsampled image with a single color component for each pixel. The green pixels are sampled at a higher rate because human eye is more sensitive to the green color than to the blue and red colors. To reconstruct a full color image from the raw data, color interpolation (also known as demosaicking) is required to estimate the two missing color components of each pixel.

The most straightforward color interpolation algorithm is bilinear interpolation, which determines the missing colors of each pixel by averaging the color values of nearby pixels. However, it suffers from artifacts such as false color and blurring especially in image areas with dense edges. This type of artifacts is also known as the zipper effect and is caused by alias - a situation where the Nyquist rates of the high spatial frequency bands of such image areas exceed the sampling rate of the CFA. As illustrated in Fig. 2, the artifacts become more profound for sharper edges because the energy of the high frequency bands increases as the edges become sharper.

Many techniques [2]-[24] have been proposed to mitigate the alias effect. One class of techniques exploits the high correlation between different color channels and transforms the

Manuscript received November 6, 2006; revised February 10, 2007. This work was supported in part by Avisonic Technology Company, Hsin-Chu, Taiwan, R.O.C. This paper was recommended by Associate Editor S. Takamura.

H.-A. Chang was with the Department of Electrical Engineering, National Taiwan University, Taipei 10617, Taiwan, R.O.C. He is now with the Computer Science and Artificial Intelligence Laboratory, Massachusetts Institute of Technology, Cambridge, MA 02142 USA.

H. H. Chen is with the Department of Electrical Engineering, Graduate Institute of Communication Engineering, and Graduate Institute of Networking and Multimedia, National Taiwan University, Taipei 10617, Taiwan, R.O.C. (e-mail: homer@cc.ee.ntu.edu.tw).

Color versions of some or all figures available online at http://ieeexplore.ieee. org.

Digital Object Identifier 10.1109/TCSVT.2007.897471

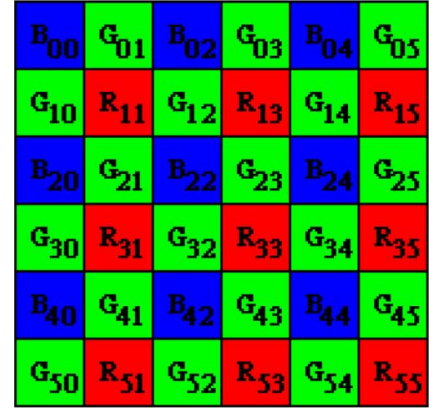

Fig. 1. Bayer color array.

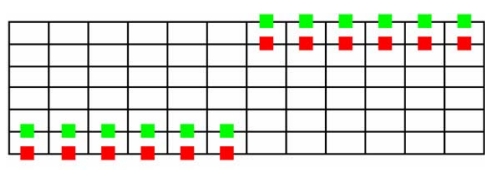

(a)

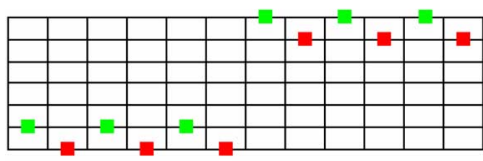

(b)

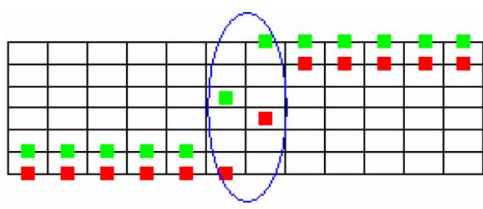

(c)

Fig. 2. Illustration of the zipper effect along a scan line of an image. Only green and red pixels are shown (modified from [6]). Vertical axis represents the color intensity. (a) Original image. (b) Bayer pattern image. (c) Image obtained by bilinear interpolation suffers from the zipper effect near the edge.

image to a new color space where the image intensity field varies more slowly. For example, Cok [2] found that the hue of pixels, namely $R / G$ or $B / G$, has a slower variation than $R G B$ and proposed a color interpolation method over hues. Pei et al. [4] found that the color differences $G-R$ and $G-B$ also exhibit slowly varying characteristics and proposed a color difference based interpolation method. However, these methods are unable to handle images where the color values of different channels do not change coherently and hence sharp edge may remain after the color space transformation.

Another important class of aliasing reduction techniques tries to solve the problem by edge-adaptive interpolation. The main idea is to adjust the weight for interpolation by using edge indicators along various edge directions. An edge indicator along an edge direction estimates the likelihood of edge crossing when moving from the pixel to be interpolated to another pixel in the 
edge direction. The weight for interpolation is inversely proportional to the likelihood estimate to prevent interpolating across an edge.

Gradient-based interpolation [20] is a simple version of edge-adaptive interpolation. It uses the vertical and horizontal gradients as the edge indicators and avoids pixels in the direction of large gradient in the interpolation. This method can be regarded as a two-directional, hard-decision edge adaptive method. The edge-adaptive interpolation can be further refined by taking more edge directions into account and by applying a soft-decision weighting policy [22]. The C2D2 method proposed by Kêhtarnavaz et al. [5] considers eight directions and uses the reciprocal of the directional derivatives as the weights for interpolation and achieves significant improvement over the gradient method.

The success of the C2D2 method suggests that the performance of edge-adaptive interpolation depends on its edge sensing ability and the robustness of its adaptive weighting policy. Motivated by this observation, we develop a stochastic approach to color interpolation. Specifically, the image is modeled as a 2-D locally stationary Gaussian process and the interpolation as stochastic estimation. Each missing color of a pixel is interpolated from a set of uniformly oriented candidate pixels based on the edge indicators and the observable data of the candidates. The relative weight of the estimate for each candidate is stochastically determined according to a reliability measure that is computed based on the statistics of the corresponding edge indicator. Then, the missing color is obtained by computing the weighted average of the estimates. Experimental results show that the algorithm proposed here can effectively eliminate the occurrence of perceptible artifact experienced in previous methods.

The rest of the paper is organized as follows. In Section II, the properties of a locally stationary Gaussian process are described. The details of the proposed algorithm, including the calculation of edge indicators and the stochastic weighting policy, are described in Section III. Experimental results and discussions are given in Section IV, followed by a summary of the paper in Section V.

\section{Locally Stationary Gaussian Process}

We begin this section by introducing the autocorrelation function of a locally stationary Gaussian process and expressing the difference of two samples in terms of the autocorrelation function. Then, we show how to normalize the differences of samples to so that they can be mapped into an identical Gaussian distribution for further probabilistic analysis.

Within a locally stationary region, the autocorrelation function $\Omega(d)$ of an image intensity field [3] can be expressed as

$$
\Omega(d)=\exp ^{\left(-d^{2} / \rho^{2}\right)}
$$

where $d$ is the distance between the two pixels considered for the function, and $\rho$ is an image-dependent parameter whose value can change from one locally stationary region to another within an image. This model implies that the power spectral density of the intensity field within a locally stationary region is also a Gaussian distribution and in accordance with the fact that the high frequency bands possess much less energy than the low frequency bands. Such high frequency bands occupy only a small portion of the image spectrum, but they are the one that causes the notorious perceptible aliasing.

For two samples of the process, $X\left(t_{1}\right)$ and $X\left(t_{2}\right)$, belonging to a locally stationary region and with $\left|t_{1}-t_{2}\right|=d$, the difference $X\left(t_{1}\right)-X\left(t_{2}\right)$ is a zero-mean Gaussian random valuable. Because of the stationary property, the standard deviation of $X\left(t_{1}\right)-X\left(t_{2}\right)$ is the same as that of $X(d)-X(0)$. Denote the standard deviation by $\sigma_{d}$; we have (2), shown at the bottom of the page, where $\mu_{X}$ and $\sigma_{X}$, respectively, are the mean and standard deviation of the process. For convenience, we denote $X(d)-X(0)$ by $\operatorname{Diff}(d)$.

Consider a set of $N$ differences $\left\{\operatorname{Diff}\left(d_{n}\right), 1 \leqslant n \leqslant N\right\}$. All these differences are Gaussian with zero mean, but depending on $d_{n}$ their standard deviations may be different. By setting a unit distance $d$ and multiplying $\operatorname{Diff}\left(d_{n}\right)$ by a normalization factor $\kappa_{n}$ as

$$
\begin{aligned}
\kappa_{n} & =\frac{\sigma_{X} \sqrt{2(1-\Omega(d))}}{\sigma_{X} \sqrt{2\left(1-\Omega\left(d_{n}\right)\right)}}=\frac{\sqrt{(1-\Omega(d))}}{\sqrt{\left(1-\Omega\left(d_{n}\right)\right)}} \\
& =\frac{\sqrt{(1-\Omega(d))}}{\sqrt{\left(1-\left(\Omega(d) d_{n}^{2} / d^{2}\right)\right.}}
\end{aligned}
$$

where the autocorrelation function expressed in (1) is used in the derivation, we can map Diff $\left(d_{n}\right)$ into a Gaussian distribution with zero mean and standard deviation $\sigma_{d}$.

Note that the normalization factor $\kappa_{n}$ is a slowly varying number when the value of $\Omega(d)$ is close to 1 . To see this, let us consider the case where $d=2$, and $d_{n}=2 \sqrt{5}$. As $\Omega(d)$ varies from 0.85 to $0.95, \kappa_{n}$ changes from 0.519 to 0.472 , representing only a $7 \%$ change. In this case, $\kappa_{n}$ can be approximated by a constant 0.5 to simplify the computation.

\section{STOCHASTIC COLOR INTERPOLATION}

The proposed interpolation algorithm is divided into three steps as shown in Fig. 3. In the first step, the missing green components of blue and red pixels are interpolated. Next, the missing red components of blue pixels and the missing blue components of red pixels are interpolated. Finally, the missing blue and red components of green pixels are interpolated. The missing green components are interpolated first because the $G$ channel has more samples available in the Bayer pattern and

$$
\begin{aligned}
\sigma_{d} & =\sqrt{E\left[(X(d)-X(0))^{2}\right]} \\
& =\sqrt{E\left[\left(\left(X(d)-\mu_{X}\right)-\left(X(0)-\mu_{X}\right)\right)^{2}\right]} \\
& =\sqrt{E\left[\left(X(d)-\mu_{X}\right)^{2}\right]+E\left[\left(X(0)-\mu_{X}\right)^{2}\right]-2 E\left[\left(X(d)-\mu_{X}\right)\left(X(0)-\mu_{X}\right)\right]} \\
& =\sigma_{X} \sqrt{2(1-\Omega(d))},
\end{aligned}
$$




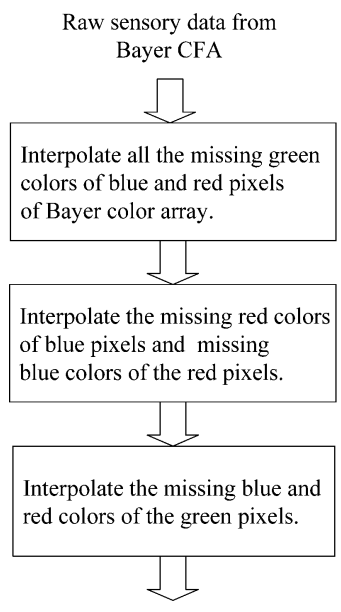

Fig. 3. Flowchart of the stochastic color interpolation algorithm.

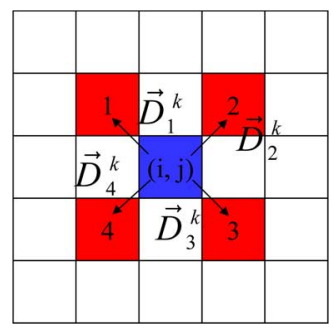

Fig. 4. Example of interpolation with four condidate pixels.

hence can generate more reliable estimate than the other two channels. A reliable estimation of the green components also helps enhance the interpolation of red and blue components because of the high correlation between these channels. In this algorithm we separate the interpolation of $R$ and from that of $B$ because the numbers of candidate pixels for these two components are different.

In this section, we describe the edge indicators and the stochastic weighting policy and show the interpolation formula used in each step of the algorithm. The index $k$ refers to the step number (which can be 1,2, or 3) in the following discussion.

\section{A. Edge Indicators}

Suppose we want to interpolate a missing color $S$ (representing $R, G$, or $B$ ) of the pixel located at $(i, j)$ (meaning $i$ th row and $j$ th column) of the Bayer pattern from $N_{k}$ candidate pixels. Denote the set of displacements (in terms of pixels) of these candidates with respect to $(i, j)$ by $\left\{\vec{D}_{n}^{k}=\left(v_{n}^{k}, h_{n}^{k}\right), 1 \leqq n \leqq N_{k}\right\}$, where $v_{n}^{k}$ is the vertical displacement from $(i, j)$ to the $n$th candidate pixel and $h_{n}^{k}$ the horizontal displacement. Fig. 4 shows an example of these notations for the interpolation of red color $(S=R)$. In this example, $N_{k}=4,\left(v_{1}^{k}, h_{1}^{k}\right)=(-1,-1),\left(v_{2}^{k}, h_{2}^{k}\right)=(-1,1)$, $\left(v_{3}^{k}, h_{3}^{k}\right)=(1,1)$, and $\left(v_{4}^{k}, h_{4}^{k}\right)=(1,-1)$.

The candidate pixels considered in the interpolation algorithm have the following properties.

1) They have the $S$ color component obtained from either the original CFA or the previous steps.

2) They are symmetric. That is, if the pixel at $\left(i+v_{n}^{k}, j+h_{n}^{k}\right)$ is a candidate, the pixel at $\left(i-v_{n}^{k}, j-h_{n}^{k}\right)$ is also a candidate.
3) For each $n$, the pixel at $\left(i+2 v_{n}^{k}, j+2 h_{n}^{k}\right)$ has the same color as the pixel at $(i, j)$.

Define the unit distance $d=2 \sqrt{\left(v_{1}^{k}\right)^{2}+\left(h_{1}^{k}\right)^{2}}$ and the $n$th edge indicator $E_{n}^{k}(i, j)$ as

$$
\begin{array}{r}
E_{n}^{k}(i, j)=0.5 \kappa_{n}\left(\left|C\left(i+v_{n}^{k}, j+h_{n}^{k}\right)-C\left(i-v_{n}^{k}, j-h_{n}^{k}\right)\right|\right. \\
\left.+\left|C\left(i+2 v_{n}^{k}, j+2 h_{n}^{k}\right)-C(i, j)\right|\right)
\end{array}
$$

where $C(i, j)$ denotes the value of CFA sensory data at $(i, j)$, and $\kappa_{n}$ is the value obtained from (3) with $d_{n}=2 \sqrt{\left(v_{n}^{k}\right)^{2}+\left(h_{n}^{k}\right)^{2}}$. Given $d$ and $d_{n}$, the value of $\kappa_{n}$ as mentioned in Section II can be approximated by a constant to save computation. Then the distributions of $E_{n}^{k}(i, j)$ for all $n$ and $k$ become the same.

Note that $C(i, j)$ and $\mathrm{C}\left(i+v_{n}^{k}, j+h_{n}^{k}\right)$ in (4) are of different colors. The reason that we consider two color channels for the edge indicator is to increase the edge sensitivity. Because a sharp change in any color channel can result in a sharp edge, jointly considering two color channels can improve the edge-detecting ability.

\section{B. Stochastic Weighting Policy}

In step $k$, the missing color $x^{k}(i, j)$ is estimated by

$$
\hat{x}^{k}(i, j)=\sum_{n=1}^{N_{k}} x_{n}^{k}(i, j) w_{n}^{k}(i, j)
$$

where $x_{n}^{k}(i, j)$ is an estimate of $x^{k}(i, j)$ based on the data available at $\left(i+v_{n}^{k}, j+h_{n}^{k}\right)$, and $w_{n}^{k}(i, j)$ is the weight associated with $x_{n}^{k}(i, j)$.

The weight $w_{n}^{k}(i, j)$ is computed according to the reliability of $x_{n}^{k}(i, j)$, which is the probability of $x_{n}^{k}(i, j)$ being a reliable estimate. Conceptually, we can consider that the reliability of $x_{n}^{k}(i, j)$ is measured with respect to a reference edge at the current pixel $(i, j)$. Denote the reference edge by $e$ and its magnitude by $|Z|$, which is the absolute difference of two pixels separated by one unit distance across the edge. If the value of the edge indicator $E_{n}^{k}(i, j)$ is larger than $|Z|$, it implies that there is an edge sharper than $e$ between the current pixel $(i, j)$ and the candidate pixel $\left(i+v_{n}^{k}, j+h_{n}^{k}\right)$. Therefore, the estimate $x_{n}^{k}(i, j)$ is unreliable. On the other hand, if $E_{n}^{k}(i, j)<$ $|Z|, x_{n}^{k}(i, j)$ is a potentially reliable estimate. With this concept in mind and under the stochastic framework discussed so far, we treat $|Z|$ as a random variable. We compute the probability $P\left(|Z|>E_{n}^{k}(i, j)\right)$ and use it as the reliability of the estimate $x_{n}^{k}(i, j)$. The weight $w_{n}^{k}(i, j)$ for $x_{n}^{k}(i, j)$ is assigned in proportion to the reliability of $x_{n}^{k}(i, j)$; hence it is proportional to $P\left(|Z|>E_{n}^{k}(i, j)\right)$. The task now is to estimate the distribution of $|Z|$ based on the information we have, namely, the values of the edge-indicators around $(i, j)$.

With the image modeled as a locally stationary Gaussian process, $Z$ becomes a zero-mean Gaussian with standard deviation $\sigma_{d}$. Therefore, the cumulative distribution function (cdf) of $|Z|$ is

$$
P(|Z| \leqslant t)=\int_{0}^{t} \frac{2}{\sqrt{2 \pi} \sigma_{d}} e^{\frac{-z^{2}}{2 \sigma_{d}^{2}}} d z
$$


The weight $w_{n}^{k}(i, j)$ for $x_{n}^{k}(i, j)$ is proportional to the reliability of $x_{n}^{k}(i, j)$ and thus satisfies the following two conditions:

$$
\begin{aligned}
w_{n}^{k}(i, j) \propto P\left(|Z|>E_{n}^{k}(i, j)\right) & =\int_{E_{n}^{k}(i, j)}^{\infty} \frac{2}{\sqrt{2 \pi} \sigma_{d}} e^{\frac{-z^{2}}{2 \sigma_{d}^{2}}} d z \\
\sum_{n=1}^{N_{k}} w_{n}^{k}(i, j) & =1 .
\end{aligned}
$$

To calculate the weight, we need to find the value of $\sigma_{d}$ first. Instead of calculating $\sigma_{d}$ directly, we can find its value from the expectance of $|Z|$ because

$$
\begin{aligned}
\operatorname{Exp}[|Z|] & =\int_{0}^{\infty} z \frac{2}{\sqrt{2 \pi} \sigma_{d}} e^{\frac{-z^{2}}{2 \sigma_{d}^{2}}} d z \\
& =\frac{2}{\sqrt{2 \pi} \sigma_{d}}\left(\sigma_{d}^{2}\right)\left(-\left.e^{\frac{-z^{2}}{2 \sigma_{d}^{2}}}\right|_{0} ^{\infty}\right) \\
& =\sqrt{\frac{2}{\pi}} \sigma_{d}
\end{aligned}
$$

which is proportional to $\sigma_{d}$. Once the expectance of $|Z|$ is known, $\sigma_{d}$ can be determined immediately. The expectance of $|Z|$ can be easily calculated from the edge indicators $E_{n}^{k}(i, j)$. Due to the locally stationary property of the image model, the difference values $\kappa_{n}\left|C\left(i+v_{n}^{k}, j+h_{n}^{k}\right)-C\left(i-v_{n}^{k}, j-h_{n}^{k}\right)\right|$ and $\kappa_{n}\left|C(i, j)-C\left(i+2 v_{n}^{k}, j+2 h_{n}^{k}\right)\right|$ used for calculating the edge indicators defined in (4) have the same distribution as $|Z|$; thus the expectance of the average of these difference values equals the expectance of $|Z|$. That is

$$
\operatorname{Exp}\left[\frac{1}{N_{k}} \sum_{n=1}^{N_{k}} E_{n}^{k}(i, j)\right]=\operatorname{Exp}[|Z|]=\sqrt{\frac{2}{\pi}} \sigma_{d} .
$$

Since the average is an unbiased estimate of the expectance, the value of $\sigma_{d}$ is estimated by averaging the edge indicator directly.

Because the integration in (7) is not in closed form, we precompute the weights and store them in a look-up table to achieve computational efficiency. Given a specified step size $\delta y$, we calculate the numerical value of

$$
f\left(y_{m}\right)=2\left(1-\Phi\left(y_{m}\right)\right)=\int_{y_{m}}^{\infty} \frac{2}{\sqrt{2 \pi}} e^{-\frac{u^{2}}{2}} d u
$$

where $y_{m}=m \cdot \delta y$ and $\Phi\left(y_{m}\right)$ is the cdf of standard Gaussian distribution, and store it in the $m$ th entry of the table. The probability $P\left(|Z|>E_{n}^{k}(i, j)\right)$ in (7) can be expressed as

$$
\begin{aligned}
P\left(|Z|>E_{n}^{k}(i, j)\right) & =\int_{E_{n}^{k}(i, j)}^{\infty} \frac{2}{\sqrt{2 \pi} \sigma_{d}} e^{-\frac{z^{2}}{2 \sigma_{d}^{2}}} d z \\
& =\int_{E_{n}^{k}(i, j) / \sigma_{d}}^{\infty} \frac{2}{\sqrt{2 \pi}} e^{-\frac{u^{2}}{2}} d u \\
& =f\left(E_{n}^{k}(i, j) / \sigma_{d}\right) \\
& =f\left(\sqrt{2 / \pi} E_{n}^{k}(i, j) / \mu\right)
\end{aligned}
$$

where $\mu$ is the average of the edge indicators

$$
\mu=\sum_{n=1}^{N_{k}} E_{n}^{k}(i, j) / N_{k}
$$

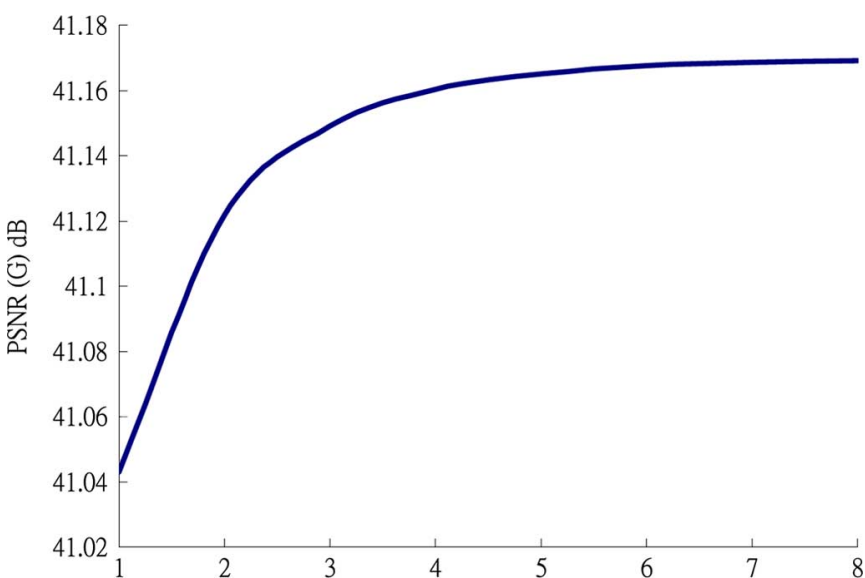

Fig. 5. Performance of the algorithm under different step sizes.

TABLE I

LOOK-UP TABLE OF $f\left(y_{m}\right)$ WITH $\left(y_{m}\right)=m \cdot 2^{-4} \sqrt{2 / \pi}$

\begin{tabular}{cccccc}
\hline \hline$m$ & $f\left(y_{m}\right)$ & $m$ & $f\left(y_{m}\right)$ & $m$ & $f\left(y_{m}\right)$ \\
\hline 1 & 0.960202 & 23 & 0.251362 & 45 & 0.024790 \\
2 & 0.920502 & 24 & 0.231326 & 46 & 0.021754 \\
3 & 0.881000 & 25 & 0.212486 & 47 & 0.019052 \\
4 & 0.841870 & 26 & 0.194746 & 48 & 0.016642 \\
5 & 0.803050 & 27 & 0.178118 & 49 & 0.014504 \\
6 & 0.764710 & 28 & 0.162600 & 50 & 0.012614 \\
7 & 0.727012 & 29 & 0.148096 & 51 & 0.010942 \\
8 & 0.689890 & 30 & 0.134600 & 52 & 0.009470 \\
9 & 0.653500 & 31 & 0.122076 & 53 & 0.008178 \\
10 & 0.617986 & 32 & 0.110504 & 54 & 0.007044 \\
11 & 0.583274 & 33 & 0.099796 & 55 & 0.006052 \\
12 & 0.549500 & 34 & 0.089932 & 56 & 0.005190 \\
13 & 0.516784 & 35 & 0.080884 & 57 & 0.004436 \\
14 & 0.485044 & 36 & 0.072574 & 58 & 0.003784 \\
15 & 0.454390 & 37 & 0.064976 & 59 & 0.003220 \\
16 & 0.424918 & 38 & 0.058060 & 60 & 0.002732 \\
17 & 0.396538 & 39 & 0.051754 & 61 & 0.002310 \\
18 & 0.369332 & 40 & 0.046030 & 62 & 0.001950 \\
19 & 0.343370 & 41 & 0.040860 & 63 & 0.001640 \\
20 & 0.318554 & 42 & 0.036180 & 64 & 0.001376 \\
21 & 0.294944 & 43 & 0.031964 & & \\
22 & 0.272580 & 44 & 0.028184 & & \\
\hline \hline
\end{tabular}

By setting $\delta y=2^{-r} \sqrt{2 / \pi}$, we can find a suitable index $\hat{m}$ for $E_{n}^{k}(i, j)$ such that

$$
\hat{m} \leqslant 2^{r} E_{n}^{k}(i, j) / \mu<\hat{m}+1 .
$$

Then, we can use $f\left(y_{\hat{m}}\right)$ to approximate $P\left(|Z|>E_{n}^{k}(i, j)\right)$.

The effect of $\delta y$ on the performance of the algorithm is investigated by varying $\delta y$ from $2^{-1} \sqrt{2 / \pi}$ to $2^{-8} \sqrt{2 / \pi}$ (i.e., from $r=1$ to $r=8$ ) and measuring the PSNR performance of the algorithm. The result is shown in Fig. 5. It can be seen that the performance of the algorithm significantly improves as $r$ increases but eventually saturates. The saturation of performance results from the fact that $\mu$ is also a random variable. If the variation of $\mu$ exceeds the step size, any further reduction of the step size can no longer provide more precise weights. Since the saturation starts roughly at $r=4$, we choose $\delta y=2^{-4} \sqrt{2 / \pi}$ and construct a table for $f\left(y_{m}\right)$, as shown in Table I. This table is used to determine the interpolation weights in the experiments described in Section IV. 


\begin{tabular}{|l|l|l|l|l|l|l|l|l|}
\hline$B_{00}$ & $G_{01}$ & $B_{02}$ & $G_{03}$ & $B_{04}$ & $G_{05}$ & $B_{06}$ & $G_{07}$ & $B_{08}$ \\
\hline$G_{10}$ & $R_{11}$ & $G_{12}$ & $R_{13}$ & $G_{14}$ & $R_{15}$ & $G_{16}$ & $R_{17}$ & $G_{18}$ \\
\hline$B_{20}$ & $G_{21}$ & $B_{22}$ & $G_{23}$ & $B_{24}$ & $G_{25}$ & $B_{26}$ & $G_{27}$ & $B_{28}$ \\
\hline$G_{30}$ & $R_{31}$ & $G_{32}$ & $R_{33}$ & $G_{34}$ & $7 R_{35}$ & $G_{36}$ & $R_{37}$ & $G_{38}$ \\
\hline$B_{40}$ & $G_{41}$ & $B_{42}$ & $G_{43}$ & $B_{443}$ & $G_{45}$ & $B_{46}$ & $G_{47}$ & $B_{48}$ \\
\hline$G_{50}$ & $R_{51}$ & $G_{52}$ & $R_{53}$ & $G_{54}$ & $R_{55}^{9}$ & $G_{56}$ & $R_{57}$ & $G_{58}$ \\
\hline$B_{60}$ & $G_{61}$ & $B_{62}$ & $G_{63}$ & $B_{64}$ & $G_{65}$ & $B_{66}$ & $G_{67}$ & $B_{68}$ \\
\hline$G_{70}$ & $R_{71}$ & $G_{72}$ & $R_{73}$ & $G_{74}$ & $R_{75}$ & $G_{76}$ & $R_{77}$ & $G_{78}$ \\
\hline$B_{80}$ & $G_{81}$ & $B_{82}$ & $G_{83}$ & $B_{84}$ & $G_{85}$ & $B_{86}$ & $G_{87}$ & $B_{88}$ \\
\hline
\end{tabular}

Fig. 6. Candidate pixels for interpolating the missing $G$ at $B_{44}$.

TABLE II

DisPlaCEMENTS FROM CURRENT PIXEL TO CANDIDATES IN STEP I

\begin{tabular}{ccc|ccc}
\hline \hline$n$ & $v_{n}^{l}$ & $h_{n}^{l}$ & $n$ & $v_{n}^{I}$ & $h_{n}^{l}$ \\
\hline 1 & 0 & -1 & 7 & -2 & +1 \\
2 & -1 & 0 & 8 & -1 & +2 \\
3 & 0 & +1 & 9 & +1 & +2 \\
4 & +1 & 0 & 10 & +2 & +1 \\
5 & -1 & -2 & 11 & +2 & -1 \\
6 & -2 & -1 & 12 & +1 & -2 \\
\hline \hline
\end{tabular}

The stochastic weighting and missing color estimation procedure of is summarized as follows.

1) Calculate $\mu$ as defined in (13).

2) For each edge indicator $E_{n}^{k}(i, j)$, find index $\hat{m}$ such that condition (14) is satisfied.

3) Temporarily set $w_{n}^{k}(i, j)=f\left(y_{\hat{m}}\right)$. Note that at this point the weights thus obtained do not satisfy condition (8) yet. The normalization is done in the next step.

4) Estimate the missing color $x^{k}(i, j)$ by

$$
\hat{x}^{k}(i, j)=\sum_{n=1}^{N_{k}} \frac{x_{n}^{k}(i, j) w_{n}^{k}(i, j)}{\sum_{n=1}^{N_{k}} w_{n}^{k}(i, j)} .
$$

\section{Color Interpolation}

Now we describe the details of the proposed algorithm. To exploit the high correlation between the $R, G$, and $B$ channels, the color difference technique described in [4] is adopted in the algorithm.

1) Interpolate the Missing $G$ of $R$ and B Pixels: In this step, the missing $G$ component of a pixel (e.g., $B_{44}$ in Fig. 6 ) is interpolated from twelve nearby neighbors of the pixel. The vertical and horizontal positions of these twelve candidates relative to the pixel to be interpolated are specified in Table II. The edge indicator of each candidate pixel is obtained by setting $d=$ $2 \sqrt{\left(v_{1}^{1}\right)^{2}+\left(h_{1}^{1}\right)^{2}}=2$ and substituting $k=1, N_{k}=12$, and

$$
\kappa_{n}= \begin{cases}1, & 1 \leqslant n \leqslant 4 \\ 0.5, & 5 \leqslant n \leqslant 12\end{cases}
$$

into (4). Here, $\kappa_{n}$ is set to 0.5 for $n=5, \ldots, 12$ to achieve the computational efficiency described in Section II. The color difference value of a blue pixel at $(i, j)$ is estimated from its $n$th candidate by

$$
b_{n}^{1}(i, j)=G\left(i+v_{n}^{1}, j+h_{n}^{1}\right)-\widehat{B}\left(i+v_{n}^{1}, j+h_{n}^{1}\right)
$$

TABLE III

Moves From CuRRent PiXel to Candidates IN STEP II

\begin{tabular}{cccccc}
\hline \hline$n$ & $v_{n}^{2}$ & $h^{2}{ }_{n}$ & $n$ & $v_{n}^{2}$ & $h^{2}{ }_{n}$ \\
\hline 1 & -1 & -1 & 3 & +1 & +1 \\
2 & -1 & +1 & 4 & +1 & -1 \\
\hline \hline
\end{tabular}

TABLE IV

Displacements From CurRent PiXel to Candidates IN SteP III

\begin{tabular}{ccc|ccc}
\hline \hline$n$ & $v_{n}^{3}$ & $h_{n}^{3}$ & $n$ & $v_{n}^{3}$ & $v_{n}^{3}$ \\
\hline 1 & 0 & -1 & 7 & -2 & +1 \\
2 & -1 & 0 & 8 & -1 & +2 \\
3 & 0 & +1 & 9 & +1 & +2 \\
4 & +1 & 0 & 10 & +2 & +1 \\
5 & -1 & -2 & 11 & +2 & -1 \\
6 & -2 & -1 & 12 & +1 & -2 \\
\hline \hline
\end{tabular}

where $\widehat{B}\left(i+v_{n}^{1}, j+h_{n}^{1}\right)$ is the average of the two blue pixels right next to $G\left(i+v_{n}^{1}, j+h_{n}^{1}\right)$. As an example, in Fig. $6, \widehat{B}(2,3)=$ $(B(2,2)+B(2,4)) / 2$ and $b_{6}^{1}(4,4)=G(2,3)-\widehat{B}(2,3)$.

The missing $G$ value of the blue pixel is interpolated by

$$
G(i, j)=B(i, j)+\frac{\sum_{n=1}^{12} w_{n}^{1}(i, j) b_{n}^{1}(i, j)}{\sum_{n=1}^{12} w_{n}^{1}(i, j)}
$$

where the weights $w_{n}^{1}(i, j)$ are obtained by the method described in Section III-B.

Similarly, the missing $G$ value of a red pixel located at $(i, j)$ is interpolated by

$$
G(i, j)=R(i, j)+\frac{\sum_{n=1}^{12} w_{n}^{1}(i, j) r_{n}^{1}(i, j)}{\sum_{n=1}^{12} w_{n}^{1}(i, j)}
$$

where $r_{n}^{1}(i, j)$ denotes the color difference value of the red pixel estimated from the $n$th candidate pixel

$$
r_{n}^{1}(i, j)=G\left(i+v_{n}^{1}, j+h_{n}^{1}\right)-\widehat{R}\left(i+v_{n}^{1}, j+h_{n}^{1}\right) .
$$

The $G$ values thus obtained are used to calculate the color difference values in the following steps.

2) Interpolate the Missing $R(B)$ of $B(R)$ Pixels: In this step, the missing color of a pixel is interpolated from the four diagonal neighbors of the pixel. The vertical and horizontal positions of these four candidates relative to the pixel to be interpolated are specified in Table III.

Because the distance from the pixel to each candidate is the same, the edge indicators are calculated by substituting $\kappa_{n}=$ $1, k=2$, and $N_{k}=4$ into (4). Given $G\left(i+v_{n}^{2}, j+h_{n}^{2}\right)$ obtained in Step 1, the color difference values are computed by

$$
\begin{aligned}
& b_{n}^{2}(i, j)=G\left(i+v_{n}^{2}, j+h_{n}^{2}\right)-B\left(i+v_{n}^{2}, j+h_{n}^{2}\right) \\
& r_{n}^{2}(i, j)=G\left(i+v_{n}^{2}, j+h_{n}^{2}\right)-R\left(i+v_{n}^{2}, j+h_{n}^{2}\right)
\end{aligned}
$$

and the weight for each candidate is computed using the method described in Section III-B. The missing $B$ and $R$ are interpolated by

$$
\begin{aligned}
& B(i, j)=G(i, j)-\frac{\sum_{n=1}^{4} w_{n}^{2}(i, j) b_{n}^{2}(i, j)}{\sum_{n=1}^{4} w_{n}^{2}(i, j)} \\
& R(i, j)=G(i, j)-\frac{\sum_{n=1}^{4} w_{n}^{2}(i, j) r_{n}^{2}(i, j)}{\sum_{n=1}^{4} w_{n}^{2}(i, j)} .
\end{aligned}
$$




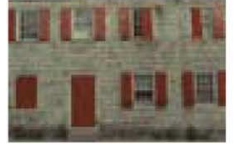

I001

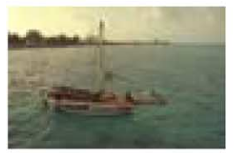

I006

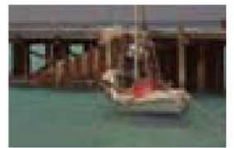

I011

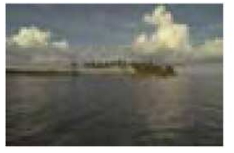

I016

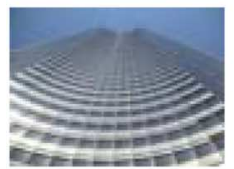

I021

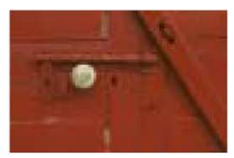

$\mathrm{I} 002$

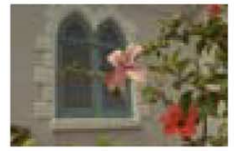

$\mathrm{I} 007$

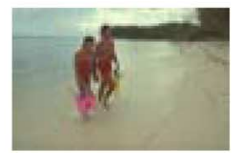

$\mathrm{I} 012$

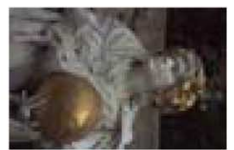

I017

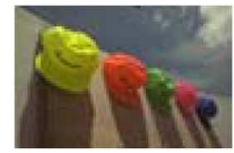

I003

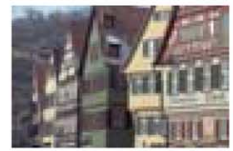

$\mathrm{I} 008$

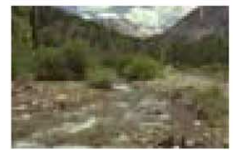

I013

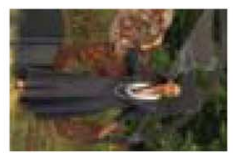

I018

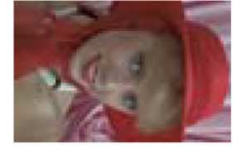

I004

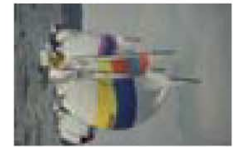

I009

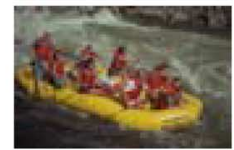

I014

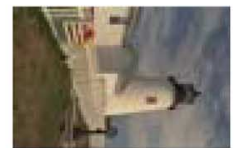

I019

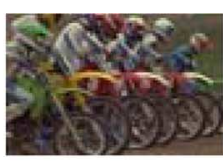

I005

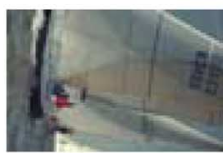

$\mathrm{I} 010$

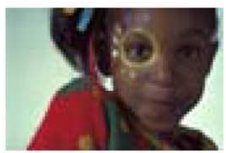

I015

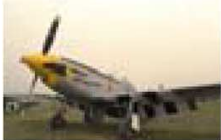

I020

Fig. 7. Test images.

3) Interpolate the Missing $R$ and $B$ of $G$ Pixels: In this step, each missing color of the pixel is interpolated from twelve candidates specified in Table IV. The edge indicators are obtained by substituting $k=3, N_{k}=12$, and $\kappa_{n}$ defined in (16) into (4). The color difference values are obtained by

$$
\begin{aligned}
& b_{n}^{3}(i, j)=G\left(i+v_{n}^{3}, j+h_{n}^{3}\right)-B\left(i+v_{n}^{3}, j+h_{n}^{3}\right) \\
& r_{n}^{3}(i, j)=G\left(i+v_{n}^{3}, j+h_{n}^{3}\right)-R\left(i+v_{n}^{3}, j+h_{n}^{3}\right) .
\end{aligned}
$$

Then the missing colors are interpolated by

$$
\begin{aligned}
& B(i, j)=G(i, j)-\frac{\sum_{n=1}^{12} w_{n}^{3}(i, j) b_{n}^{3}(i, j)}{\sum_{n=1}^{12} w_{n}^{3}(i, j)} \\
& R(i, j)=G(i, j)-\frac{\sum_{n=1}^{12} w_{n}^{3}(i, j) r_{n}^{3}(i, j)}{\sum_{n=1}^{12} w_{n}^{3}(i, j)} .
\end{aligned}
$$

\section{EXPERIMENTS AND DISCUSSION}

The test images used in our experiments consist of $20 \mathrm{im}$ ages (labeled I001-I020) from the Kodak image database and a building image (I021) that has fine, dense edges in most areas of the image. These full-color test images, shown in Fig. 7, were first down-sampled to the Bayer pattern and then interpolated to full color. The performance of the various interpolation methods are measured in terms of peak signal-to-noise ratios (PSNRs) of the $R, G$, and $B$ channels, mean square error (MSE), and normalized color difference (NCD). The first two metrics are performed in the RGB space, while the last one is in the CIELAB color space.

The PSNR of the $G$ channel of an image with height $H$ and width $W$ is obtained by

$$
\operatorname{PSNR}_{G}=10 \cdot \log _{10}\left(\frac{255^{2} \cdot H \cdot W}{\sum_{i=1}^{H} \sum_{j=1}^{W}\left(G(i, j)-G^{\prime}(i, j)\right)^{2}}\right)
$$

where $G(i, j)$ and $G^{\prime}(i, j)$ represent the green components of the original and the interpolated images, respectively. The PSNRs of the $R$ and $B$ channels are calculated in a similar way.

The MSE of the interpolated image is given by

$$
\begin{aligned}
\mathrm{MSE}=\frac{1}{3 \cdot H \cdot W} \sum_{i=1}^{H} \sum_{j=1}^{W} & \left((\Delta R(i, j))^{2}\right. \\
& \left.+(\Delta G(i, j))^{2}+(\Delta B(i, j))^{2}\right)
\end{aligned}
$$

where $\Delta$ denotes the difference between the original color value and the interpolated color value.

The NCD of the interpolated image is computed according to the formula shown in (31) at the bottom of the page. Because the square root operation is performed before the summation in

$$
\mathrm{NCD}=\frac{\sum_{i=1}^{H} \sum_{j=1}^{W}\left(\sqrt{(\Delta L(i, j))^{2}+(\Delta a(i, j))^{2}+(\Delta b(i, j))^{2}}\right)}{\sum_{i=1}^{H} \sum_{j=1}^{W}\left(\sqrt{(L(i, j))^{2}+(a(i, j))^{2}+(b(i, j))^{2}}\right)} .
$$


TABLE V

COLOR PSNR OF THE INTERPOLATED IMAGES

\begin{tabular}{|c|c|c|c|c|c|c|c|c|c|c|c|c|c|c|c|c|c|c|}
\hline \multirow{2}{*}{$\begin{array}{c}\text { Image } \\
\text { No. }\end{array}$} & \multicolumn{3}{|c|}{ GB } & \multicolumn{3}{|c|}{$\mathrm{CDB}$} & \multicolumn{3}{|c|}{ C2D2 } & \multicolumn{3}{|c|}{$\overline{\mathrm{AH}}$} & \multicolumn{3}{|c|}{ Proposed } & \multicolumn{3}{|c|}{ Proposed with MF } \\
\hline & SNR_B & & & & & & $\mathrm{P}$ & & & & & & $D^{2}$ & & & & & \\
\hline $\mathrm{I} 001$ & 3 & 4 & .800 & & 75 & 992 & & 5 & 4.272 & .934 & 36.902 & 34.598 & 3 & 39. & 0 & 0 & 11 & \\
\hline & & & & & & & & & & & & & & & & 6 & 0 & \\
\hline & & & & & & & & & & & & & & & & & & \\
\hline & & 6 & & & 42.622 & & & & & & 42 & & & & & & 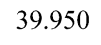 & 58 \\
\hline & & 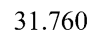 & & & 78 & & & & & & & & & & & & 9 & .726 \\
\hline & & & & & & & & & & & & & & & & & & \\
\hline 7 & & .054 & & & 43.020 & & & & & & 4 & & & & & & & $.3 \%$ \\
\hline & & & & & 21 & & & & & & & & & & & & & \\
\hline & & & & & & & & & & & & & & & & & 4 & \\
\hline 0 & & 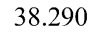 & & & 5 & & & & & & & & & & & & 7 & 213 \\
\hline & & & & & 78 & & & & & & 39 & & & & & & & 90 \\
\hline & & & & & & & & & & & & & & 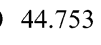 & & & 0 & \\
\hline & & .021 & & & 3 & & & & & & 32 & & & 3 & & & 4 & 16 \\
\hline & & ta & & & 9 & & & 3 & & & 38 & & & & & & & 249 \\
\hline & & & & & 42 & & & & & & & & & & & & 6 & \\
\hline & & 3 & & & 2 & & & & & & 4 & & & 0 & & & 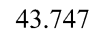 & \\
\hline Ic & & .463 & & & 41.505 & & & & & & 41 & & & 43 & & 9 & 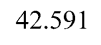 & .350 \\
\hline & & & & & & & & & & & & & & 5 & & & 1 & .50 \\
\hline & & & & & 38.265 & 22 & & & & & 40 & & & & & & 5 & 7.329 \\
\hline & & 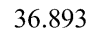 & & & 41.439 & & & & & & 41.3 & 00 & & 42 & & 0 & 41.9 & 8.406 \\
\hline & & 5.34 & & & 30.213 & 69 & & 28. & & & 31.134 & & 95 & 32 & 79 & 30.425 & 33.095 & 9.290 \\
\hline & 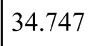 & 35.210 & ז & 34.970 & ו & 33.330 & 6.846 & 37.875 & 36.656 & 38.197 & 40.166 & 51.5 & SO. & 41.100 & 36.996 & 0.000 & 70.02 & 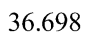 \\
\hline
\end{tabular}

Original Data: $\{10,10,10,10,2,2,2,2\}$

Estimated Data $1:\{11,12,11,11,1,1,1,1\}$

Estimated Data 2: $\{11,11,13,11,1,2,2,3\}$

\begin{tabular}{|c|c|c|}
\hline & MSE & NCD \\
\hline Data 1 & 1.375 & 0.1875 \\
\hline Data 2 & 1.75 & 0.1667 \\
\hline
\end{tabular}

Fig. 8. Example of the inconsistency between MSE and NCD.

this equation, the NCD metric may not necessarily be consistent with MSE and PSNR. An example of the inconsistency is illustrated in Fig. 8, where the MSE of the first estimated data set is smaller than that of the second one, but the NCD gives the opposite result.

The conversion from RGB to CIELAB space may also contribute to the inconsistency, since it is not an orthogonal operation. Generally, if interpolation method A leads to a smaller MSE but larger NCD than method B for an image, the interpolation error of the image generated by A tends to have smaller variance than that by $\mathrm{B}$, implying that $\mathrm{A}$ is more stable. Because the proposed algorithm is based on the locally stationary assumption, it is more region-independent than the other methods and hence is able to achieve minimal MSE.

The stochastic interpolation algorithm proposed in this paper is compared against four existing methods: gradient-based interpolation (GB), color-difference-based interpolation (CDB) [4], CFA interpolation using correlations and directional Derivatives
(C2D2) [5], and adaptive homogeneity-directed interpolation (AH) [8]. The GB, CDB, and C2D2 methods are described in Section I. To investigate the effect of median filtering on the performance of the proposed algorithm, a $3 \times 3$ median filter (MF) [14] is applied as a post processing operation, and the result is compared.

The AH method proposed by Hirakawa et al. [8] applies a five point 1-D filter to the raw sensory data to generate a vertically interpolated image and a horizontally interpolated image. Then the homogeneity of each pixel of the two images is calculated. At each $(i, j)$, the pixel from the image with the larger homogeneity is chosen as the output of the interpolation. Since it has been demonstrated that the $\mathrm{AH}$ method has better performance than the Alternating Projection method [9], we only include the former in the comparison. Note also that we chose to compare against the original AH method instead of the simplified versions [8] because it has the best performance.

The PSNR values of the images interpolated by different methods are listed in Table V, whereas the MSE and NCD values are provided in Table VI. As we can see, the AH method and the proposed algorithm have the best performance among all methods. Furthermore, the average performance of the proposed algorithm is better than that of $\mathrm{AH}$ by about $1 \mathrm{~dB}$ in PSNR_G and $13 \%$ in MSE. However, AH has a slightly better NCD. This probably can be attributed to the fact that AH uses a criterion similar to NCD in calculating the homogeneity.

Another interesting observation based on the average performance is that the median filter does not bring improvement to the interpolation. By examining the interpolated images carefully, however, we find that images with regions of dense edges 
TABLE VI

MSE AND NCD OF INTERPOLATED IMAGES

\begin{tabular}{|c|c|c|c|c|c|c|c|c|c|c|c|c|}
\hline \multirow{2}{*}{$\begin{array}{c}\text { Image } \\
\text { No. }\end{array}$} & \multicolumn{2}{|c|}{ GB } & \multicolumn{2}{|c|}{$\mathrm{CDB}$} & \multicolumn{2}{|c|}{$\mathrm{C} 2 \mathrm{D} 2$} & \multicolumn{2}{|c|}{$\mathrm{AH}$} & \multicolumn{2}{|c|}{ Proposed } & \multicolumn{2}{|c|}{ Proposed with MF } \\
\hline & MSE & $\operatorname{NCD}(\%)$ & MSE & $\operatorname{NCD}(\%)$ & MSE & $\operatorname{NCD}(\%)$ & MSE & $\operatorname{NCD}(\%)$ & MSE & $\mathrm{NCD}(\%)$ & MSE & $\mathrm{NCD}(\%)$ \\
\hline I001 & 40.89 & 3.329 & 24.23 & 2.692 & 23.57 & 2.558 & 18.90 & 2.280 & 10.79 & 1.856 & 10.39 & 1.769 \\
\hline I002 & 12.31 & 2.018 & 59.40 & 4.158 & 8.22 & 1.775 & 7.03 & 1.618 & 8.56 & 2.011 & 9.91 & 2.143 \\
\hline I003 & 8.01 & 1.252 & 21.47 & 1.857 & 5.23 & 1.105 & 3.11 & 0.887 & 4.78 & 1.217 & 6.68 & 1.359 \\
\hline I004 & 11.12 & 1.741 & 18.02 & 2.052 & 6.39 & 1.411 & 6.06 & 1.328 & 7.47 & 1.603 & 9.65 & 1.792 \\
\hline I005 & 41.81 & 3.931 & 24.23 & 3.221 & 20.18 & 2.888 & 13.99 & 2.446 & 12.64 & 2.515 & 15.79 & 2.685 \\
\hline I006 & 29.81 & 2.048 & 19.34 & 1.856 & 20.22 & 1.771 & 9.47 & 1.214 & 9.81 & 1.366 & 8.13 & 1.274 \\
\hline I007 & 8.38 & 1.203 & 39.05 & 2.904 & 4.76 & 1.034 & 4.15 & 0.962 & 5.71 & 1.302 & 7.91 & 1.467 \\
\hline I008 & 56.70 & 3.101 & 43.47 & 3.018 & 39.23 & 2.742 & 26.58 & 2.197 & 25.83 & 2.343 & 22.66 & 2.243 \\
\hline I009 & 9.05 & 1.189 & 8.37 & 1.089 & 5.53 & 0.962 & 4.05 & 0.860 & 4.09 & 0.830 & 5.10 & 0.874 \\
\hline I010 & 10.58 & 1.309 & 7.78 & 1.284 & 6.02 & 1.046 & 4.20 & 0.936 & 4.18 & 0.943 & 5.06 & 1.023 \\
\hline I011 & 24.75 & 2.574 & 16.58 & 2.348 & 14.93 & 2.184 & 10.67 & 1.754 & 9.00 & 1.923 & 9.22 & 1.939 \\
\hline I012 & 7.61 & 0.899 & 8.10 & 0.924 & 5.23 & 0.780 & 3.27 & 0.637 & 4.01 & 0.729 & 5.08 & 0.762 \\
\hline I013 & 107.31 & 5.333 & 40.71 & 3.399 & 55.99 & 3.832 & 43.14 & 3.470 & 17.95 & 2.327 & 14.20 & 2.093 \\
\hline I014 & 27.52 & 2.783 & 27.48 & 2.502 & 16.38 & 2.186 & 13.97 & 1.958 & 17.17 & 2.110 & 22.53 & 2.277 \\
\hline I015 & 14.68 & 1.709 & 19.81 & 1.939 & 8.72 & 1.410 & 8.09 & 1.315 & 7.70 & 1.417 & 10.27 & 1.584 \\
\hline I016 & 13.00 & 1.738 & 10.25 & 1.666 & 9.76 & 1.559 & 3.94 & 1.076 & 5.61 & 1.335 & 4.96 & 1.311 \\
\hline I017 & 14.89 & 2.190 & 7.25 & 1.649 & 7.83 & 1.700 & 6.51 & 1.556 & 5.07 & 1.618 & 5.31 & 1.692 \\
\hline I018 & 39.82 & 4.164 & 33.75 & 4.135 & 21.74 & 3.072 & 19.03 & 2.980 & 12.00 & 2.621 & 13.08 & 2.776 \\
\hline I019 & 18.50 & 1.928 & 20.33 & 1.996 & 12.54 & 1.628 & 8.43 & 1.374 & 10.44 & 1.530 & 9.73 & 1.518 \\
\hline I020 & 14.05 & 0.981 & 10.54 & 0.948 & 8.25 & 0.832 & 6.85 & 0.775 & 6.19 & 0.891 & 6.97 & 0.919 \\
\hline I021 & 169.71 & 4.088 & 109.75 & 4.158 & 97.94 & 3.321 & 69.20 & 2.634 & 60.94 & 2.831 & 55.81 & 2.715 \\
\hline Avg. & 32.40 & 2.358 & 27.14 & 9.724 & 18.98 & 1.895 & 13.84 & 1.631 & 11.90 & 1.682 & 12.31 & 1.725 \\
\hline
\end{tabular}

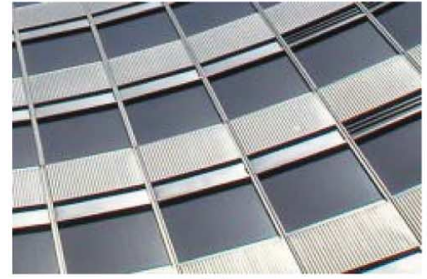

(a)

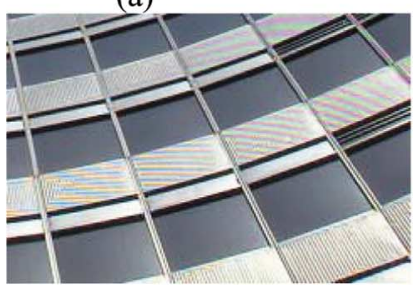

(e)

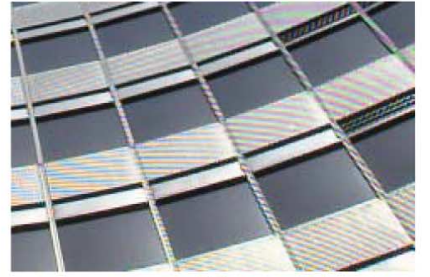

(b)

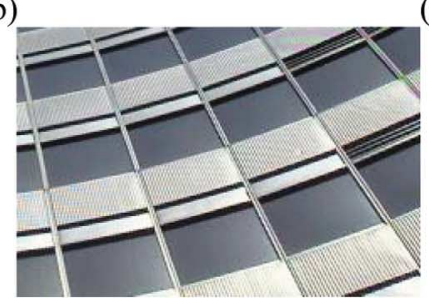

(f)

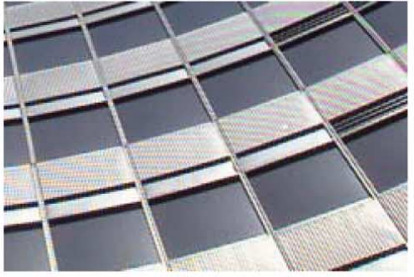

(c)

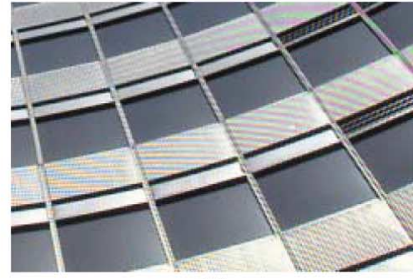

(d)

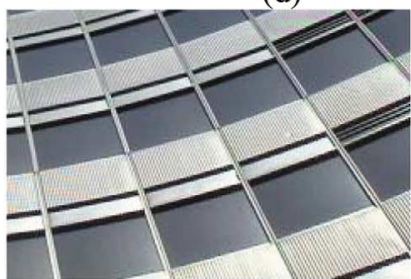

(g)

Fig. 9. Part of the building image (I021). (a) Original. (b) GB. (c) CDB. (d) C2D2. (e) AH. (f) Proposed. (g) Proposed with MF.

such as the building image (I021), the light house image (I019), and the brook image (I013) do benefit from the media filtering. For images with large smooth areas, the median filter tends to degrade the overall performance of the interpolation, though it is able to remove the aliasing near the edges.

The robustness of the proposed algorithm against aliasing is demonstrated in Figs. 9-12. It is evident from Fig. 9 that the proposed algorithm is able to deal with dense edges and reduce the perceptible aliasing effectively. Similar fine results can be observed at the sticker near the center of Fig. 10, the wires and trees in Fig. 11, and the slogan and mascot in Fig. 12.
The results show that the algorithm produces better quality for the interpolated images by preserving the sharpness of object boundaries and by effectively preventing the occurrences of false colors.

The proposed algorithm provides significant performance improvement without additional computational costs. Although it needs to compute the average of the edge indicators and to perform table look-up, it does not need to compute the reciprocal of the edge-indicator as the C2D2 algorithm does. The overall computational cost of the proposed algorithm is similar to that of other soft-decision edge-adaptive methods. 


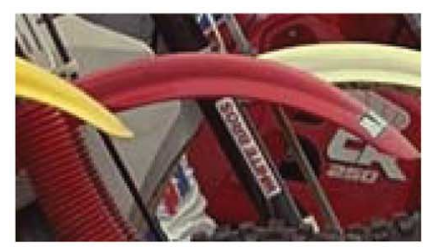

(a)

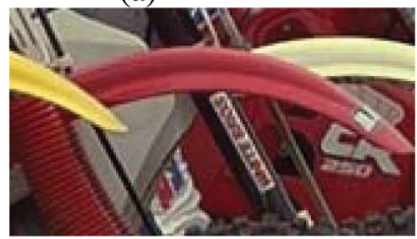

(e)

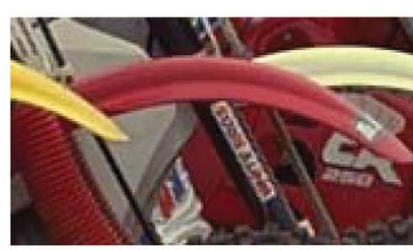

(b)

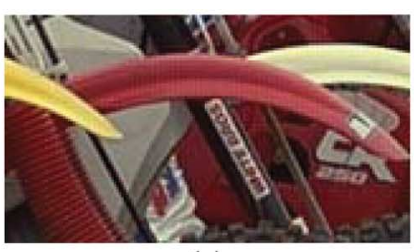

(c)

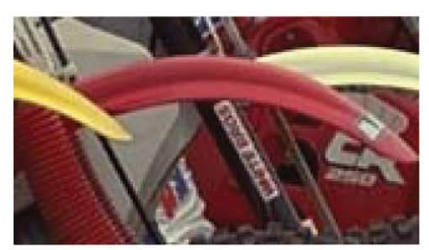

(d)

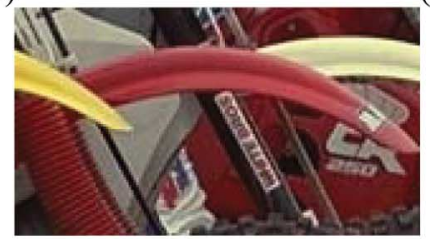

(f)

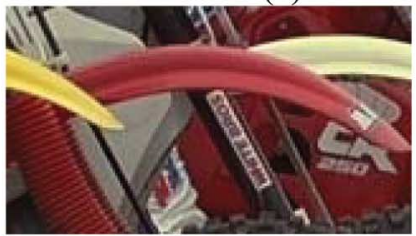

(g)

Fig. 10. Part of the motors image (I005). (a) Original.(b) GB.(c) CDB.(d) C2D2.(e) AH.(f) Proposed.(g) Proposed with MF

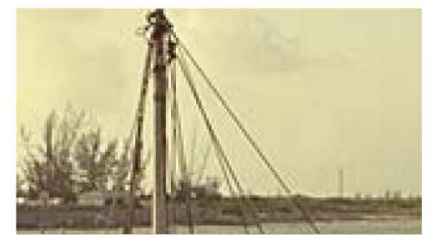

(a)

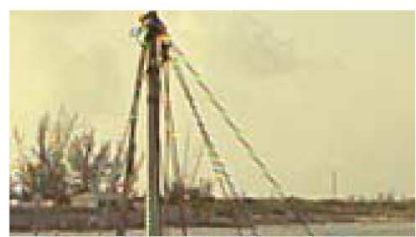

(b)

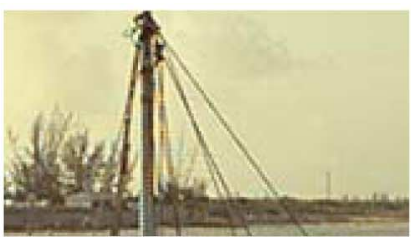

(c)

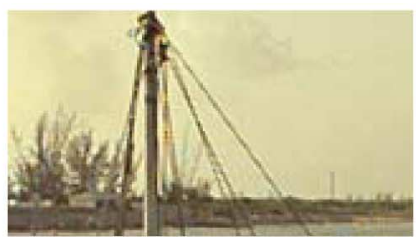

(d)

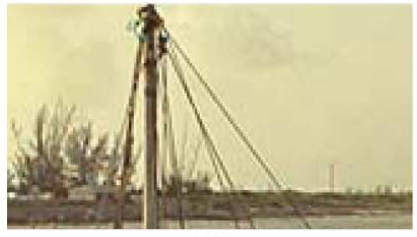

(e)

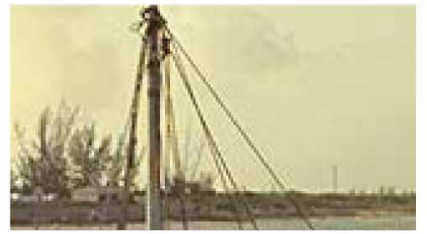

(f)

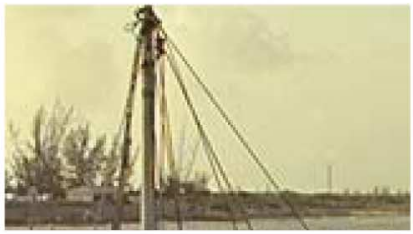

(g)

Fig. 11. Part of the sailboat image (I006). (a) Original.(b) GB.(c) CDB.(d) C2D2.(e) AH.(f) Proposed.(g) Proposed with MF.

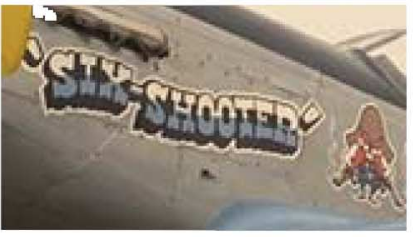

(a)

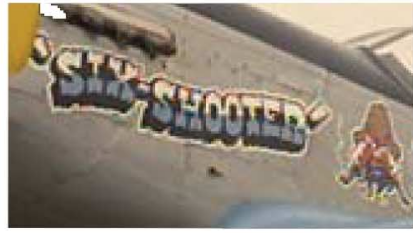

(b)

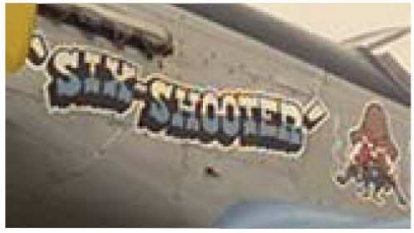

(c)

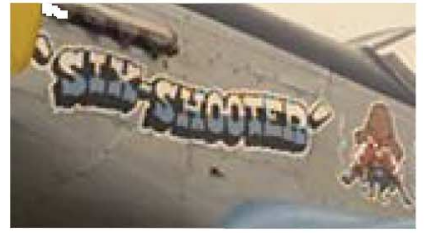

(d)

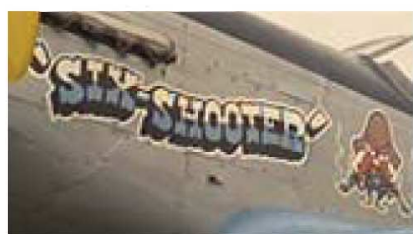

(e)

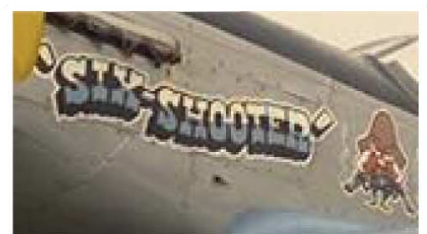

(f)

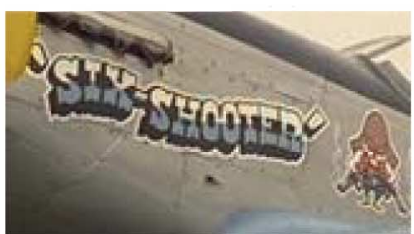

(g)

Fig. 12. Part of the airplane image (I020). (a) Original. (b) GB. (c) CDB. (d) C2D2. (e) AH. (f) Proposed. (g) Proposed with MF.

\section{SUMMARY}

In this paper, we have presented a stochastic estimation algorithm for adaptive color interpolation of the Bayer pattern. Each missing color of a pixel is interpolated from a set of uniformly oriented candidate pixels. By using the properties of the image under the locally stationary Gaussian process assumption and a stochastic weighting policy, the algorithm demonstrates high edge sensing ability and robust weighting against aliasing.

\section{REFERENCES}

[1] B. E. Bayer, “Imaging array,” U.S. Patent 3971 065, Jul. 20, 1976.

[2] D. R. Cok, "Signal processing method and apparatus for producing interpolated chrominance values in sampled color image signal," U.S. Patent 4 642 678, 1987.

[3] X. Li and M. T. Orchard, "New edge-directed interpolation," IEEE Trans. Image Process., vol. 10, no. 10, pp. 1521-1527, Oct. 2001.

[4] S. C. Pei and I. K. Tam, "Effective color interpolation in CCD color filter arrays using signal correlation," IEEE Trans. Circuits Syst. Video Technol., vol. 13, no. 6, pp. 503-513, Jun. 2003. 
[5] N. Kehtarnavaz, H. Oh, and Y. Yoo, "Color filter array interpolation using correlations and directional derivatives," J. Electron. Imag., vol. 12, no. 4, pp. 621-632, Oct. 2003.

[6] R. Ramanath, W. E. Synder, and G. L. Bilbro, "Demosiacking methods for Bayer color array," J. Electron. Imag., vol. 11, no. 3, pp. 306-315, Jul. 2002.

[7] B. S. Hur and M. G. Kang, "High definition color interpolation scheme for progressive scan CCD image sensor," IEEE Trans. Consum. Electron., vol. 47, no. 1, pp. 179-186, Feb. 2001.

[8] K. Hirakawa and T. W. Parks, "Adaptive homogeneity-directed demosaicing algorithm," IEEE Trans. Image Process., vol. 14, no. 3, Mar. 2005.

[9] B. K. Gunturk, Y. Altunbasak, and R. M. Mersereau, "Color plane interpolation using alternating projections," IEEE Trans. Image Process., vol. 11, no. 9, pp. 997-1013, Sep. 2002.

[10] R. Ramanath and W. E. Snyder, "Adaptive demosaicking," J. Electron. Imag., vol. 12, no. 4, pp. 633-642, Oct. 2003.

[11] W. Lu and Y. P. Tan, "Color filter array demosaicking: New method and performance measure," IEEE Trans. Image Process., vol. 12, no. 10, pp. 1194-1210, Oct. 2003.

[12] R. Lukac, K. N. Plataniotis, D. Hatzinakos, and M. Aleksic, "A novel cost effective demosaicing approach," IEEE Trans. Consum. Electron., vol. 50, no. 1, pp. 256-261, Feb. 2004.

[13] R. Lukac and K. N. Plataniotis, "Data adaptive filters for demosaicking: A framework," IEEE Trans. Consum. Electron., vol. 51, no. 2, pp. 560-570, May 2005.

[14] T. W. Freeman, "Median Filter for Reconstructing Missing Color Samples," U.S. Patent 4724 395, 1988.

[15] B. K. Gunturk, J. Glotzbach, Y. Altunbasak, R. W. Schafer, and R. M. Mersereau, "Demosaicking: Color filter array interpolation," IEEE Signal Process. Mag., vol. 22, no. 1, pp. 44-54, Jan. 2005.

[16] R. Lukac, K. Martin, and K. N. Plataniotis, "Demosaicked image processing using local color ratios," IEEE Trans. Circuits Syst. Video Technol., vol. 14, no. 6, pp. 914-920, Jun. 2004.

[17] R. Lukac and K. N. Plataniotis, "Normalized color ratio modeling for CFA interpolation," IEEE Trans. Consum. Electron., vol. 50, no. 2, pp. 737-745, May 2004.

[18] X. Wu and N. Zhang, "Primary-consistent soft-decision color demosaicking for digital cameras," IEEE Trans. Image Process., vol. 13, no. 9, pp. 1263-1274, Sep. 2004.

[19] D. Alleysson, S. Stisstrunk, and J. Hérault, "Linear demosaicing inspired by the human visual system," IEEE Trans. Image Process., vol. 14, no. 4, pp. 439-449, Apr. 2005.

[20] C. A. Laroche and M. A. Prescott, "Apparatus and method for adaptively interpolating a full color image utilizing chrominance gradients," U.S. Patent 5373 322, Dec. 12, 1994.

[21] J. F. Hamilton, Jr. and J. E. Adams Jr., "Adaptive color plane interpolation in single sensor color electronic camera," U.S. Patent 5 629 734, May 13, 1997.
[22] E. Chang, S. Cheung, and D. Pan, "Color filter array recovery using a threshold-based variable number of gradients," Proc. SPIE, vol. 3650 , pp. 36-43, Mar. 1999.

[23] R. Kimmel, "Demosaicing: Image reconstruction from color CCD samples," IEEE Trans. Image Process., vol. 8, no. 9, pp. 1221-1228, Sep. 1999.

[24] H. S. Malvar, L.-W. He, and R. Culter, "High-quality linear interpolation for demosaicing of Bayer-patterned color images," in Proc. ICASSP, May 2004, pp. 485-488.

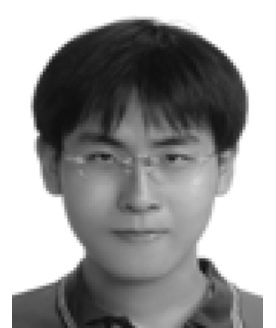

Hung-An Chang received the B.S. degree in electrical engineering from National Taiwan University, Taiwain, R.O.C., in 2004. He is currently pursuing the Ph.D. degree in the Computer Science and $\mathrm{Ar}$ tificial Intelligence Laboratory, Massachusetts Institute of Technology, Cambridge, and working on the application of digital signal processing and machine learning techniques to speech processing and spoken language understanding.

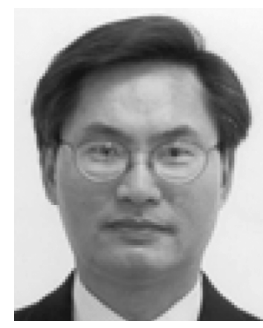

Homer H. Chen (S'83-M'86-SM'01-F'03) received the Ph.D. degree in electrical and computer engineering from University of Illinois at Urbana-Champaign.

Since August 2003, he has been with the College of Electrical Engineering and Computer Science, National Taiwan University, Taiwan, R.O.C., where he is Irving T. Ho Chair Professor. Prior to that, he had held various research and development management and engineering positions in leading US companies including AT\&T Bell Labs, Rockwell Science Center, iVast, and Digital Island over a period of 17 years. He was a US delegate of the ISO and ITU standards committees and contributed to the development of many new interactive multimedia technologies that are now part of the MPEG-4 and JPEG-2000 standards. His research interests lie in the broad area of multimedia processing and communications.

Dr. Chen is an Associate Editor of IEEE TRANSACTIONS ON CIRCUITS AND SySTEMS FOR VIDEO TECHNOLOGY. Hehas served as an Associate Editor for IEEE TRANSACTIONS ON IMAGE PROCESSING from 1992 to 1994, Guest Editor for IEEE TRANSACTIONS ON CIRCUITS AND SYSTEMS FOR VIDEO TECHNOLOGY in 1999, and Editorial Board Member for Pattern Recognition from 1989 to 1999. 